\title{
FLUXO DIFUSIVO E BIODISPONIBILIDADE DE ZINCO, COBRE, FERRO E MANGANÊS NO SOLO: INFLUÊNCIA DA CALAGEM, TEXTURA DO SOLO E RESÍDUOS VEGETAIS ${ }^{(1)}$
}

\author{
Rodinei Facco Pegoraro ${ }^{(2,6)}$, Ivo Ribeiro da Silva ${ }^{(3)}$, Roberto Ferreira \\ Novais $^{(3,6)}$, Eduardo de Sá Mendonça ${ }^{(3,6)}$, Fabrício de Oliveira \\ Gebrim $^{(4)} \&$ Frederico Fonseca Moreira ${ }^{(5)}$
}

\begin{abstract}
Resumo
Em solos altamente intemperizados, o fluxo difusivo é o principal mecanismo de transporte de $\mathrm{Zn}, \mathrm{Cu}, \mathrm{Fe}$ e $\mathrm{Mn}$ até à superfície das raízes das plantas. Práticas de manejo, como a calagem e a adição de resíduos vegetais ao solo, podem influir no fluxo difusivo e na disponibilidade desses micronutrientes catiônicos. Neste sentido, o presente trabalho objetivou avaliar o fluxo difusivo e a biodisponibilidade de $\mathrm{Zn}$, $\mathrm{Cu}$, Fe e Mn, nas formas catiônicas ou aniônicas, em dois Latosssolos, sob influência de doses de calcário e de resíduos vegetais. Para tanto, os resíduos vegetais, do feijão guandu ou do milheto, foram incorporados em amostra de um Latossolo textura média (LVA) e outro de textura argilosa (LV). Para avaliar o fluxo difusivo, utilizou-se resina de troca aniônica (positivamente carregada) e de troca catiônica (negativamente carregada) na forma de lâminas, incubadas no solo, em câmaras de difusão, durante quinze dias. Os resultados obtidos demonstraram que a calagem reduziu o fluxo difusivo dos micronutrientes, mas a incorporação de resíduos vegetais aos solos atuou inversamente, minimizando o efeito negativo da calagem na difusão. O $\mathrm{Cu}$ e o Fe foram transportados, predominantemente, na forma aniônica, enquanto o $\mathrm{Zn}$ e o Mn, predominantemente, na forma catiônica. A adição de resíduos vegetais também aumentou o pH dos solos e melhorou a absorção dos micronutrientes pelas plantas de milho, principalmente, quando se incorporou resíduo de milheto, no $L V A$, e de guandu, no $L V$.
\end{abstract}

Termos de indexação: feijão guandu, milheto, transporte, adubo verde, resina de troca iônica.

\footnotetext{
(1) Recebido para publicação em junho de 2005 e aprovado em agosto de 2006 .

${ }^{(2)}$ Doutorando do Programa de Pós-Graduação do em Solos e Nutrição de Plantas da Universidade Federal de Viçosa - UFV. Avenida P.H. Rolfs s/n., CEP 36571-000 Viçosa (MG). E-mail: rodinei_pegoraro@yahoo.com.br

(3) Professor do Departamento de Solos, UFV. E-mails: ivosilva@ufv.br; rfnovais@ufv.br; esm@ufv.br

(4) Doutorando do Programa de Pós-Graduação em Solos e Nutrição de Plantas, Departamento de Solos, UFV. E-mail: fgebrim@yahoo.com.br

(5) Estudante de Iniciação Científica, Departamento de Solos, UFV. E-mail: fredifm@bol.com.br

(6) Bolsista do CNPq.
} 


\title{
SUMMARY: DIFFUSIVE FLUXAND BIOAVAILABILITYOFMICRONUTRIENTS IN SOILS: INFLUENCE OF LIMING, SOIL TEXTURE AND GREEN MANURE
}

\begin{abstract}
In highly weathered tropical soils the diffusive flux is the main transport mechanism of $\mathrm{Zn}, \mathrm{Cu}, \mathrm{Fe}$, and $\mathrm{Mn}$ to the root surface of plants. Management practices such as liming and the presence of plant residues can alter the diffusive flux of these cationic micronutrients. The aim of the present study was to evaluate the diffusive flux in anionic and cationic forms and bioavaiability of cationic micronutrients $\mathrm{Zn}, \mathrm{Cu}, \mathrm{Fe}$ and $\mathrm{Mn}$ in Oxisols with distinct textures as influenced by liming doses, in the absence and presence of plant residues. Two plant materials widely used as green manure - guandu bean and millet - were incorporated into two Oxisols (a loamy-sand Red Yellow Latosol - LVA and a clayey Red Latosol - LV). The diffusive flux was evaluated using a positively charged anion exchange resin membrane and a negatively charged cation exchange resin membrane. The membranes were incubated with the soils for 15 days. Results showed that liming reduced the diffusive flux of the cationic micronutrients. The presence of green manures attenuated this negative effect of liming on $\mathrm{Zn}, \mathrm{Cu}, \mathrm{Fe}$ and $\mathrm{Mn}$ transport. $\mathrm{Cu}$ and $\mathrm{Fe}$ were transported predominantly in the anionic form, whereas $\mathrm{Zn}$ and $\mathrm{Mn}$ did mainly in the cationic form. The addition of green manure also raised the soil $\mathrm{pH}$, and improved the absorption of cationic micronutrient by corn plants, especially when millet was incorporated into the LVA and guandu bean into the $L V$.
\end{abstract}

Index terms: guandu bean, millet, transport, green manures, ionic resin.

\section{INTRODUÇÃO}

Em solos altamente intemperizados, a pobreza química dos constituintes do solo e a alta afinidade dos micronutrientes catiônicos ( $\mathrm{Zn}, \mathrm{Cu}, \mathrm{Fe}$ e $\mathrm{Mn}$ ) pelos colóides do solo levam, geralmente, à baixa concentração desses na solução do solo. Nesta condição, o fluxo difusivo é o principal mecanismo de transporte de $\mathrm{Zn}, \mathrm{Cu}$, Fe e Mn no solo (Barber, 1995). Várias práticas de manejo do solo podem influenciar o transporte a curta distância desses micronutrientes.

Nesses solos ácidos, a obtenção de altas produtividades de culturas exigentes passa, necessariamente, pelo emprego da calagem. No entanto, ao elevar o $\mathrm{pH}$ do solo, a calagem reduz a disponibilidade de micronutrientes cationicos, tais como: Zn e Mn (Sanders, 1983). Muitos outros trabalhos relacionam o aumento do $\mathrm{pH}$ decorrente da calagem com a deficiência de micronutrientes metálicos, por diminuir sua solubilidade na solução do solo, tornando-os menos disponíveis para as plantas (Rhoton, 2000). Caires \& Fonseca (2000), ao avaliarem a absorção de nutrientes pela soja, em experimento com doses de calcário em superfície no sistema de semeadura direta, verificaram redução de $\mathrm{Zn}$ e $\mathrm{Mn}$ absorvidos pela cultura. Caires et al. (2003) também verificaram redução nos teores foliares de Zn na soja, após três cultivos sucessivos, em decorrência do aumento do $\mathrm{pH}$ do solo proporcionado pela adição de calcário na superfície em sistema de semeadura direta e na absorção de Mn, sobretudo quando se incorporou calcário ao solo. Em condição de $\mathrm{pH}$ mais elevado, a concentração de micronutrientes catiônicos na solução do solo é reduzida com a formação de compostos de baixa solubilidade, levando ao decréscimo do seu fluxo difusivo (Barber, 1995). Evidência para tal vem de trabalho em que o aumento do $\mathrm{pH}$ do solo foi um fator de grande importância no controle do fluxo difusivo de Zn na forma catiônica, chegando a reduzi-lo até 26 vezes, estando o decréscimo diretamente associado à menor concentração desses micronutrientes na solução do solo (Oliveira et al., 1999).

A deficiência de micronutrientes é comum em regiões tropicais onde se tem observado aumento do $\mathrm{pH}$ dos solos cultivados por longos períodos de tempo, tal como na Índia. Essa baixa disponibilidade desses nutrientes seria improvável se houvesse aumento no teor da matéria orgânica do solo (Duxbury et al., 1989). Tem sido sugerido que a maior disponibilidade de resíduos vegetais, decorrente da adoção de sistemas conservacionistas, como a semeadura direta, pode aumentar o teor de matéria orgânica do solo (Sá et al., 2001; Bayer et al., 2004). Isso pode requerer uma mudança na disponibilidade de micronutrientes pelo aumento da formação de complexos solúveis com a matéria orgânica do solo e seus produtos de decomposição (Jones et al., 2003) e compostos hidrossolúveis lixiviados dos resíduos vegetais em processo de decomposição (Franchini et al., 1999). Dentre esses compostos, estão os ácidos orgânicos de baixa massa molecular (AOBMM) (Franchini et al., 2003) que são capazes de complexar os micronutrientes catiônicos do solo (Jones, 1998; Pegoraro, 2003). Embora a importância de compostos orgânicos no 
transporte de micronutrientes catiônicos seja largamente aceita (Vulkan et al., 2002; Jones et al., 2003), ainda não existe informação sobre a natureza iônica dos complexos transportados e a magnitude do efeito de resíduos vegetais sobre o fluxo difusivo de micronutrientes.

O presente trabalho objetivou avaliar o fluxo difusivo e a biodisponibilidade dos micronutrientes $\mathrm{Zn}$, $\mathrm{Cu}, \mathrm{Fe}$ e Mn, nas formas aniônicas ou catiônicas, em Latosssolos com texturas distintas, sob influência de doses de calcário e de resíduos vegetais.

\section{MATERIAL E MÉTODOS}

O experimento consistiu de um fatorial $2 \times 2 \times 4$, correspondendo a dois solos: Latossolo VermelhoAmarelo (LVA) textura média e um Latossolo Vermelho (LV) textura argilosa; dois resíduos vegetais: feijão guandu (Cajanus cajan (L.) Millsp.) e milheto (Pennisetum americanum (L) Leeke), e quatro doses de calcário. As doses de corretivo $\left(\mathrm{CaCO}_{3}\right.$ e $\mathrm{MgCO}_{3}$ na proporção molar 4:1) foram calculadas para equivaler a 0 (testemunha); 0,5; 1 e 2 vezes a dose para atingir $60 \%$ de saturação por bases (Alvarez V. \& Ribeiro, 1999), e corresponderam a $0 ; 1,23 ; 2,47$; $4,94 \mathrm{t} \mathrm{ha}^{-1}$, no LV, e a $0 ; 1,21 ; 2,43 ; 4,85 \mathrm{t} \mathrm{ha}^{-1}$, no LVA.

O LV foi coletado em área de mata nativa, no município de São Sebastião, MG. O LVA foi coletado em área de cerrado típico, no município de Três Marias, MG. As amostragens dos solos foram realizadas na camada de $0-20 \mathrm{~cm}$, secas ao ar, destorroadas, passadas em peneira com abertura de malha de $2 \mathrm{~mm}$ e homogeneizadas. Retiraram-se alíquotas para a caracterização química e física. Os resultados dessas análises no LVA e no LV foram: $\mathrm{pH}$ $\left(\mathrm{H}_{2} \mathrm{O}\right), 4,72$ e 5,06; $\mathrm{Ca}^{2+}, 0,17$ e 0,58 $\mathrm{cmol}_{\mathrm{c}} \mathrm{dm}^{-3} ; \mathrm{Mg}^{2+}$, 0,00 e $0,24 \mathrm{cmol}_{\mathrm{c}} \mathrm{dm}^{-3}\left(\mathrm{KCl} 1 \mathrm{~mol} \mathrm{~L}^{-1}\right) ; \mathrm{Zn}, 17,82 \mathrm{e}$ $16,6 \mathrm{mg} \mathrm{dm}^{-3} ; \mathrm{Cu}, 7,3$ e 10,4 $\mathrm{mg} \mathrm{dm}^{-3} ; \mathrm{Fe}, 84,1$ e 55,2 $\mathrm{mg} \mathrm{dm}^{-3}$; Mn, 8,2 e 55,2 $\mathrm{mg} \mathrm{dm}^{-3}$ (Mehlich-1); CO, 10,0 e 14,9 $\mathrm{g} \mathrm{kg}^{-1}$ (Walkley-Black) e argila, $34 \mathrm{e}$ $47 \%$, respectivamente.

Os resíduos vegetais de milheto e guandu (parte aérea de ambos) foram colhidos em estádio de crescimento vegetativo pleno, secos em estufa, por $72 \mathrm{~h}$, a $65^{\circ} \mathrm{C}$ e triturados em moinho tipo Wiley com peneira de malha de $0,5 \mathrm{~mm}$. Alíquotas foram submetidas à digestão nitroperclórica para posterior determinação dos teores totais de $\mathrm{Zn}, \mathrm{Cu}$, Fe e $\mathrm{Mn}$, por espectrofotometria de absorção atômica, que foram de: 17,$13 ; 13,47 ; 433,47$ e $52,83 \mathrm{mg} \mathrm{kg}^{-1}$, respectivamente, no feijão guandu e 23,$77 ; 8,57 ; 249,27$ e $65,97 \mathrm{mg} \mathrm{kg}^{-1}$, respectivamente, no milheto. Em ambos os resíduos vegetais, foram determinados os teores de ácidos orgânicos de baixa massa molecular (AOBMM), por meio de cromatografia de íons (Silva et al., 2001).
Após o material vegetal ter sido seco e triturado em moinho com peneira de $0,5 \mathrm{~mm}$, pesou-se $0,1 \mathrm{~g}$ do material para maceração em almofariz na presença de $\mathrm{N}$ líquido, com o intuito de favorecer a quebra do tecido vegetal. Posteriormente, o material vegetal foi macerado em $4 \mathrm{~mL}$ de água ultrapura e centrifugado (3.000 g), durante 5 min. Essa operação foi repetida quatro vezes, até que o sobrenadante não mais apresentasse coloração esverdeada. O sobrenadante foi concentrado sob vácuo e, posteriormente, as amostras foram reconstituídas em $1 \mathrm{~mL}$ de água ultrapura e passadas em filtro de membrana de 0,45 $\mu \mathrm{m}$. Antes de iniciar a análise por cromatografia, as amostras de milheto foram diluídas, novamente, 20 vezes, e as de feijão guandu, 10 vezes. O volume de amostra injetada no cromatógrafo iônico (DX 600 - Dionex, Sunnyvale, CA, EUA) foi de $120 \mu \mathrm{L}$, utilizando-se amostrador automático. Como fase estacionária, utilizou-se a coluna de troca aniônica AS-11 (Dionex). Os AOBMM foram eluidos com uma fase móvel composta de: (a) água ultrapura, (b) $\mathrm{NaOH} 10 \mathrm{mmol} \mathrm{L}^{-1}$, (c) $\mathrm{NaOH} 100 \mathrm{mmol} \mathrm{L}^{-1}$ e (d) metanol $100 \%$. Os eluentes foram mantidos fechados sob pressão constante de gás hélio para eliminar a interferência de carbonatos e evitar flutuações na linha de base. O fluxo de eluição foi de 1,3 mL min-1. Os compostos foram detectados por condutividade elétrica suprimida, utilizando-se um detector eletroquímico. A identificação dos compostos foi feita, comparando-se o tempo de retenção de cada composto com o de amostras-padrão já conhecidas. Todo o sistema cromatográfico era controlado por meio do software Chromeleon (Dionex).

Os solos foram incubados com as doses de calcário, sob condições de casa de vegetação, por um período de 15 dias e com umidade equivalente a $80 \%$ da capacidade de campo (-30 kPa). Após esse período, foram adicionados aos solos uma dose de $10 \mathrm{mg} \mathrm{dm}^{-3}$, de $\mathrm{Zn}, \mathrm{Cu}, \mathrm{Fe}$ e $\mathrm{Mn}$ na forma de sais de sulfato, e uma solução com outros macro e micronutrientes (Novais et al., 1991). Decorrido um período adicional de 15 dias de incubação, o feijão guandu ou o milheto foram incorporados aos solos em dose equivalente a $18 \mathrm{t} \mathrm{ha}^{-1}$ de matéria seca.

Para avaliar o fluxo difusivo, foram instaladas câmaras de difusão, constituídas por anéis de PVC com $100 \mathrm{~mm}$ de diâmetro e $50 \mathrm{~mm}$ de altura. Inicialmente, adicionou-se metade do solo $\left( \pm 0,2 \mathrm{dm}^{3}\right)$ no anel de PVC e sobre esta camada foram colocadas duas lâminas de resina (com $2 \mathrm{~cm}$ de largura por $5 \mathrm{~cm}$ de comprimento), uma trocadora de ânions, positivamente carregada, tipo base forte 204U2ARA (Ionics Inc., EUA), e outra trocadora de cátions, negativamente carregada, tipo ácido forte CR $61 \mathrm{CZR}$ (Ionics Inc., EUA) pré-condicionadas com $\mathrm{NaHCO}_{3}$ $1 \mathrm{~mol} \mathrm{~L}^{-1}$, por um período de $12 \mathrm{~h}$. Sobre estas, o restante do solo foi cuidadosamente acondicionado de modo a garantir bom contato. Esses dois tipos de resinas foram usados para quantificar o fluxo difusivo dos micronutrientes, tanto na forma catiônica, como na aniônica. 
$\mathrm{O}$ solo foi mantido com umidade correspondente à capacidade de campo $(-30 \mathrm{kPa})$, por meio do envolvimento de toda a coluna com filme plástico. As câmaras foram mantidas em laboratório a uma temperatura ambiente de $25 \pm 2{ }^{\circ} \mathrm{C}$. Após quinze dias de incubação, as câmaras de difusão foram desmontadas, as lâminas foram retiradas, lavadas com água deionizada, agitadas por $30 \mathrm{~min}$ em água ultrapura (milliQ) e, então, colocadas em HCl $0,5 \mathrm{~mol} \mathrm{~L}^{-1}$ por $1,5 \mathrm{~h}$ sob agitação constante $(150 \mathrm{rpm})$ para dessorção dos elementos adsorvidos na resina. A determinação de $\mathrm{Zn}, \mathrm{Fe}, \mathrm{Cu}$ e $\mathrm{Mn}$ nos extratos foi feita por espectrofotometria de absorção atômica. $\mathrm{O} \mathrm{Ca}$ e o Mg trocáveis dos solos foram extraídos com solução de KCl $1 \mathrm{~mol} \mathrm{~L}^{-1}$ e seus teores nos extratos foram determinados por espectrofotometria de absorção atômica.

Após a desmontagem das câmaras de difusão, utilizaram-se os solos com os tratamentos para realização de bioensaio com milho, durante 25 dias. Os solos foram acomodados em potes plásticos de $0,5 \mathrm{~L}$, onde foram semeadas quatro sementes de milho. Decorrida uma semana, procedeu-se ao raleio, mantendo-se uma planta por pote. Ao final deste período, foi coletada a parte aérea das plantas para quantificação da matéria seca e posterior digestão nitroperclórica para a determinação dos teores de Zn, $\mathrm{Cu}, \mathrm{Fe}$ e $\mathrm{Mn}$. Com base nesses teores e na produção de matéria seca, foram calculados os conteúdos de micronutrientes acumulados pelas plantas. Também foi calculada a dose de calcário necessária para se atingir a produção máxima de matéria seca da planta de milho (Máxima Eficiência Física - MEF).

Os resultados foram submetidos à análise de variância para modelos fatoriais, testando-se as interações entre solos x resíduos x doses de calcário e o desdobramento da interação tripla, utilizando o programa estatístico SAEG 6.0 (Sistema de Análises Estatísticas e Genéticas) e, quando pertinente, foi realizada análise de regressão.

\section{RESULTADOS E DISCUSSÃO}

\section{Fluxo difusivo dos micronutrientes catiônicos}

O fluxo difusivo médio de $\mathrm{Zn}$ no solo textura média $\left(20,4 \mathrm{nmol} \mathrm{m}^{-2} \mathrm{~s}^{-1}\right)$ na ausência de resíduos vegetais foi significativamente $(\mathrm{P} \leq € 0,05)$ maior do que o obtido no solo textura argilosa $\left(10,0 \mathrm{nmol} \mathrm{m}{ }^{-2} \mathrm{~s}^{-1}\right)$. Para $\mathrm{Cu}$, os valores de fluxo difusivo foram semelhantes para ambos os solos (9,6 e $9,9 \mathrm{nmol} \mathrm{m}{ }^{-2} \mathrm{~s}^{-1}$, para o LVA e $\mathrm{LV}$, respectivamente). Quanto ao Fe e ao Mn, obtevese maior fluxo difusivo no solo argiloso $(\mathrm{Fe}=21,2 \mathrm{e}$ $41,0 \mathrm{nmol} \mathrm{m}{ }^{-2} \mathrm{~s}^{-1}, \mathrm{Mn}=148,4$ e $274,7 \mathrm{nmol} \mathrm{m}^{-2} \mathrm{~s}^{-1}$ no LVA e LV, respectivamente). Na presença de resíduos vegetais, o fluxo difusivo médio de $\mathrm{Zn}$ e Fe foi maior no solo argiloso $(\mathrm{Zn}=10,9$ e $15,6, \mathrm{Fe}=39,9$ e $53,7 \mathrm{nmol} \mathrm{m}^{-2} \mathrm{~s}^{-1}$, no LVA e LV, respectivamente), menor para o Mn $\left(361,5\right.$ e $282,2 \mathrm{nmol} \mathrm{m}^{-2} \mathrm{~s}^{-1}$, no LVA e LV, respectivamente) e semelhante para o $\mathrm{Cu}$ nos dois solos (13,6 e 12,8 $\mathrm{nmol} \mathrm{m}^{-2} \mathrm{~s}^{-1}$, no LVA e LV, respectivamente).

O nutriente com maior fluxo difusivo nos solos foi o $\mathrm{Mn}$, seguido do $\mathrm{Fe}$, do $\mathrm{Zn}$ e por último, do $\mathrm{Cu}$. O maior transporte do Mn por difusão está associado, possivelmente, ao seu maior teor nestes solos e à maior solubilidade em relação aos outros três micronutrientes catiônicos (Barber, 1995). O Cu, graças à alta afinidade com a matéria orgânica (Temminghoff et al., 1998) e com os óxidos de $\mathrm{Fe}$ e $\mathrm{Al}$ (Korte et al., 1976), tem o seu fluxo bastante reduzido em relação aos outros micronutrientes catiônicos.

O fluxo difusivo do $\mathrm{Cu}$ e do $\mathrm{Fe}$ foi significativamente ( $\mathrm{P} \leq € 0,05)$ maior com as espécies aniônicas $(\mathrm{Cu}=9,64$ e $2,41, \mathrm{Fe}=34,88$ e $6,69 \mathrm{nmol} \mathrm{m}^{-2} \mathrm{~s}^{-1}$ para as resinas aniônicas e catiônicas, respectivamente), sugerindo que seu transporte pode ter ocorrido como complexos organo-metálicos com carga líquida negativa. No entanto, a participação de espécies aniônicas inorgânicas com $\mathrm{SO}_{4}{ }^{2-}$ não pode ser descartada. Já com o $\mathrm{Zn}$ e $\mathrm{Mn}$, as formas transportadas foram predominantemente catiônicas. Vulkan et al. (2002), estudando a especiação de $\mathrm{Cu}$ e Zn da fração solúvel de uma mistura de solo com lodo de esgoto compostado, reportaram que mais de $91 \%$ do $\mathrm{Cu}$ complexado encontrava-se sob formas aniônicas. Para o Zn, eles observaram um caráter anfótero, ligando-se a compostos catiônicos ou aniônicos, dependendo das condições do meio (teor de $\mathrm{C}$ orgânico solúvel em água e atividade iônica) e tempo de incubação.

Os resíduos vegetais depositados na superfície ou incorporados ao solo contêm muitos compostos orgânicos que, ao serem lavados pela água da chuva, juntam-se àqueles derivados da atividade microbiana do solo, favorecendo a formação de ligações com os sítios de ligação dos óxidos de $\mathrm{Fe}$ e $\mathrm{Al}$ (van Hees et al., 2003), a dissolução e solubilização dos minerais do solo (Jones, 1998) e a formação de complexos com os nutrientes que se encontram na solução do solo (Strobel, 2001; Renella et al., 2004) que podem ser transportados para a superfície das raízes e, posteriormente, absorvidos pelas plantas.

No presente estudo, após a aplicação de resíduos vegetais, obteve-se maior fluxo difusivo de $\mathrm{Zn}, \mathrm{Cu}, \mathrm{Fe}$ e $\mathrm{Mn}$, tanto para a resina aniônica (positivamente carregada), como para a resina catiônica (negativamente carregada), em ambos os solos, quando comparados aos tratamentos que receberam somente a calagem, à exceção no fluxo difusivo do $\mathrm{Zn}$ e do $\mathrm{Cu}$ para a resina catiônica, no solo textura média, onde ocorreu o contrário. O aumento do transporte desses nutrientes após a adição de resíduo vegetal ocorreu, possivelmente, em virtude do aumento da formação de complexos organo-metálicos com carga líquida negativa. De fato, as análises dos resíduos vegetais de guandu e milheto identificaram vários AOBMM, tais como: cítrico e málico, que podem complexar os micronutrientes catiônicos e vir a beneficiar o seu fluxo difusivo nos 
solos. Outro fator que pode ter contribuído para o aumento no fluxo difusivo dos micronutrientes foi a adição aos solos de $\mathrm{Zn}, \mathrm{Cu}$, Fe e $\mathrm{Mn}$ advindos do próprio resíduo vegetal incubado, muito embora esse incremento seja bastante pequeno, principalmente, de $\mathrm{Zn}, \mathrm{Cu}$ e $\mathrm{Mn}$, em relação à dose de micronutrientes adicionada aos solos $\left(10 \mathrm{mg} \mathrm{dm}^{-3}\right)$. Segundo estudos anteriores, é provável que a lixiviação de compostos hidrossolúveis do resíduo vegetal em processo de decomposição possibilite maior ciclagem de micronutrientes no solo (Franchini et al., 1999).

Embora se tenha verificado maior teor de compostos orgânicos de baixa massa molecular no resíduo de milheto (acetato: 126,9; formato: 217,9 e 312,9; butarato: 1,5 e 46,8; succinato: ND (abaixo do limite de detecção) e 72,7; malato: 37,7 e 502,2; malonato: 14,7 ; fumarato: 15,9 e 36,2 ; oxalato: 23,3 e 137,8 ; citrato: 24,9 e 27,6 ; isocitrato: ND e $34,4 \mathrm{mmol} \mathrm{kg}^{-1}$ nos resíduos de guandu e milheto, respectivamente), obteve-se maior fluxo difusivo de $\mathrm{Fe}$ para a resina aniônica no solo testado com resíduo de guandu, graças, possivelmente, ao maior conteúdo desse íon no resíduo (433,47 $\left.\mathrm{mg} \mathrm{kg}^{-1}\right)$. Adicionalmente, o fato de ser uma leguminosa faz com que o guandu decomponha mais rapidamente pela ação da biota do solo (Oliveira et al., 2002). Todavia, com o resíduo de milheto obteve-se maior fluxo difusivo de $\mathrm{Mn}$, para a resina aniônica, e de Fe e Mn para a resina catiônica. Notou-se uma maior concentração total dos ácidos orgânicos e de compostos com maior número de grupamentos carboxílicos (malato, fumarato, oxalato, citrato, isocitrato) no milheto, o que pode conferir uma maior estabilidade da ligação organo-metálica (Strobel, 2001), aumentando o teor desses nutrientes na solução do solo em formas solúveis e, conseqüentemente, do seu fluxo difusivo para as raízes das plantas em razão do aumento do gradiente de concentração.

O efeito do tipo de solo, resíduo vegetal e das doses de calcário no fluxo difusivo dos micronutrientes catiônicos podem ser observados nas figuras $1,2,3 \mathrm{e}$ 4. Dada a ocorrência de interação tripla significativa $(\mathrm{P} \leq € 0,05)$ entre os dois tipos de solos $\mathrm{x}$ dois resíduos vegetais $\mathrm{x}$ quatro doses de calagem, pode-se observar que, na ausência dos resíduos vegetais, o aumento das doses de corretivo proporcionaram redução do fluxo difusivo de $\mathrm{Zn}, \mathrm{Fe}, \mathrm{Cu}$ e $\mathrm{Mn}$, sendo esse decréscimo mais acentuado nas formas catiônicas, uma vez que, com o aumento do $\mathrm{pH}$, ocorrem hidroxilação e formação de espécies metálicas de baixa solubilidade (Lindsay, 1979; Barber, 1995). Segundo Nunes et al. (2004), o aumento do $\mathrm{pH}$ do solo por meio da calagem restringe fortemente o fluxo difusivo de $\mathrm{Fe}$ e pode até, juntamente com teores elevados de $\mathrm{P}$ disponíveis $\mathrm{e}$ déficits hídricos, causar o aparecimento de sintomas de deficiência de Fe pela absorção insatisfatória deste por plantas de café. Oliveira et al. (1999) também observaram grandes restrições ao fluxo difusivo de $\mathrm{Zn}$ após elevarem o $\mathrm{pH}$ do solo por meio da calagem.

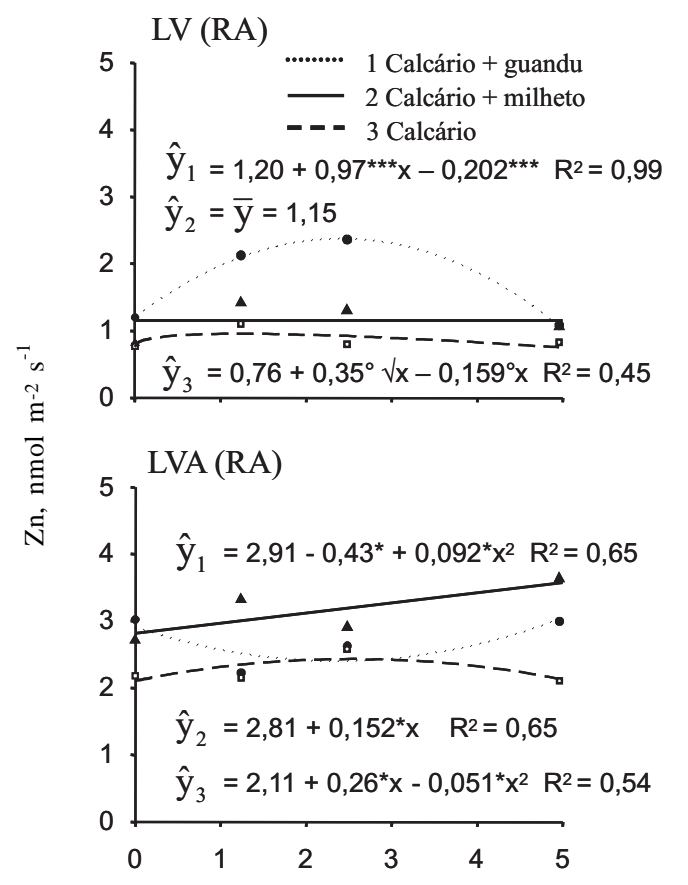

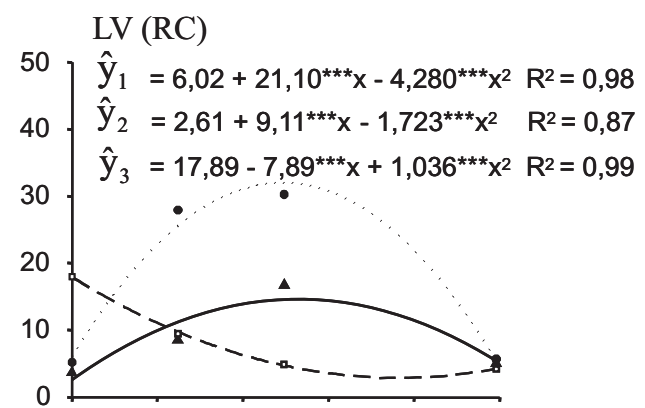

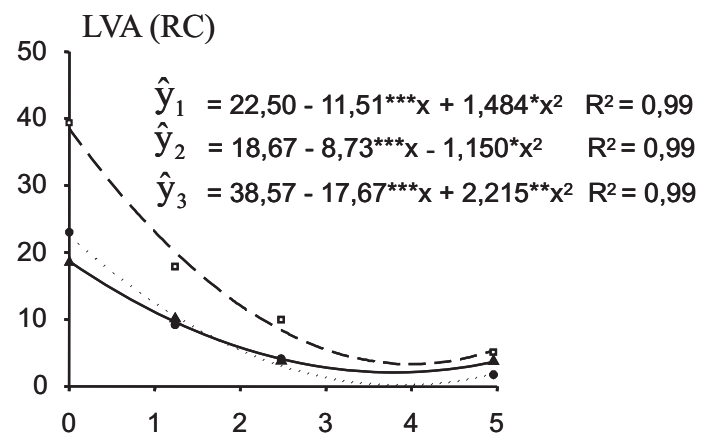

CALCÁRIO, $\mathrm{t} \mathrm{ha}^{-1}$

Figura 1. Fluxo difusivo de Zn para as resinas aniônicas (RA) e catiônicas (RC) em resposta à adição de resíduos de guandu ou milheto, com ou sem calagem, em solo textura média (LVA) e argilosa (LV). ${ }^{\text {ns }},{ }^{\circ}$, ${ }^{*},{ }^{* * * * * *}$ : não-significativo e significativos a $P<10,5,1$ e $0,1 \%$, respectivamente. 


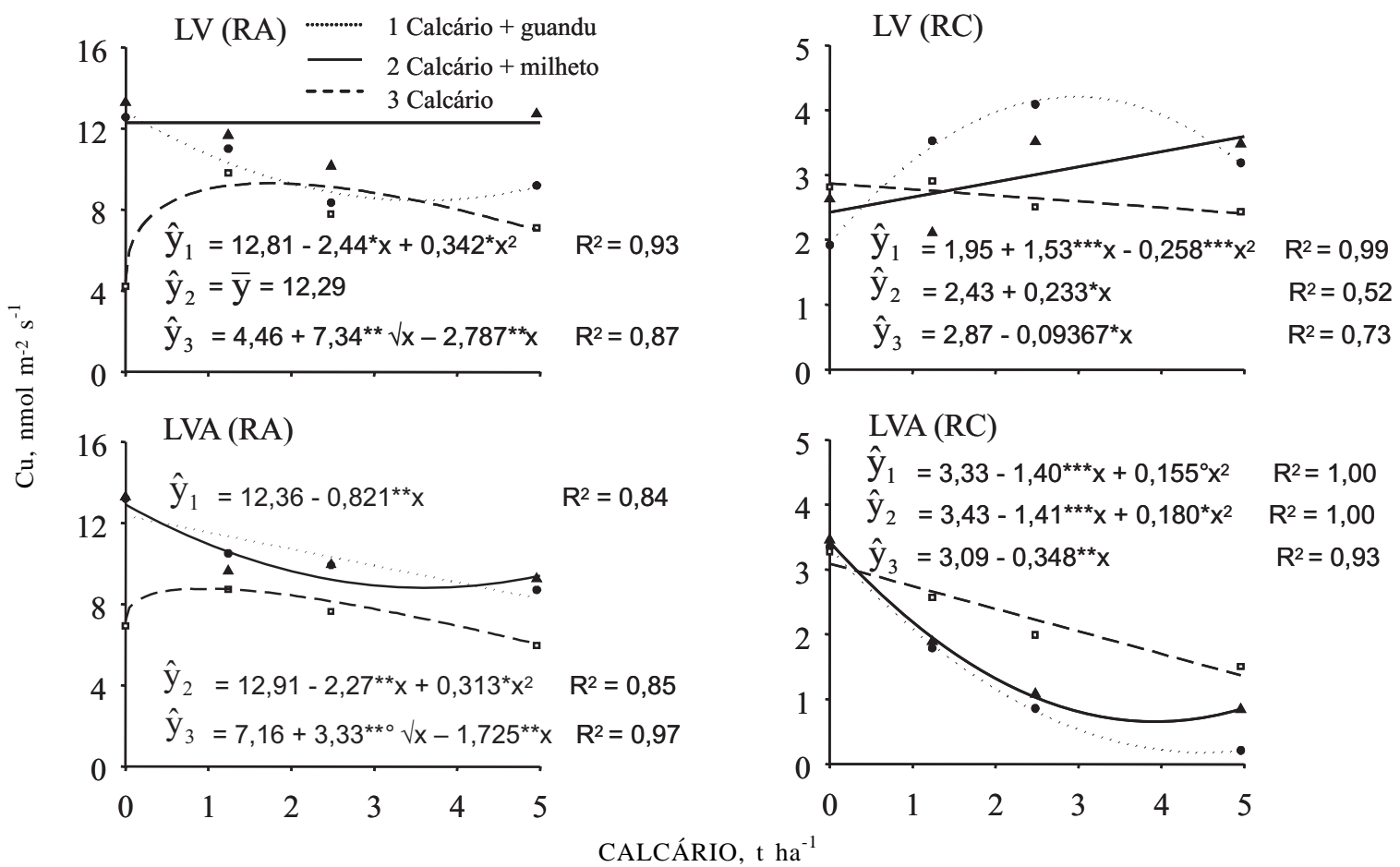

Figura 2. Fluxo difusivo de Cu para as resinas aniônicas (RA) e catiônicas (RC) em resposta à adição de resíduos de guandu ou milheto, com ou sem calagem, em solo textura média (LVA) e argilosa (LV). ${ }^{\mathrm{ns}},{ }^{\circ}$, ${ }^{*}, * * * * * *$ : não-significativo e significativos a $P<10,5,1$ e $0,1 \%$, respectivamente.

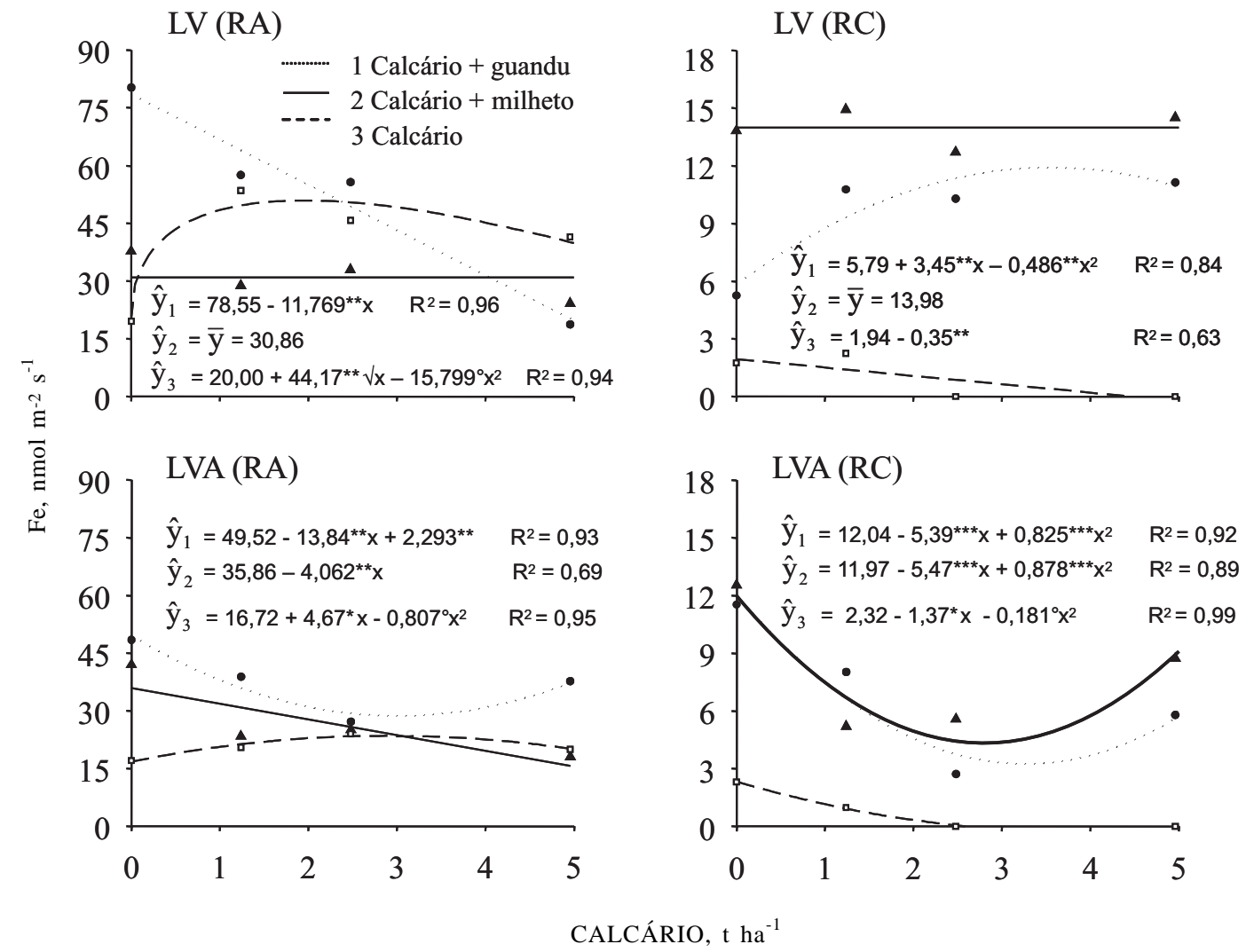

Figura 3. Fluxo difusivo de Fe para as resinas aniônicas (RA) e catiônicas (RC) em resposta à adição de resíduos vegetais de guandu ou milheto, com ou sem calagem, em solo textura média (LVA) e argilosa (LV). ${ }^{\mathrm{ns}},,^{*},{ }^{* * *},{ }^{* * * *}$ : não-significativo e significativos a $\mathrm{P}<10,5,1$ e $0,1 \%$, respectivamente. 
A adição de resíduos vegetais, de modo geral, minimizou a restrição ao fluxo difusivo dos micronutrientes ocasionada pela elevação do $\mathrm{pH}$ dos solos. Isso foi mais evidente para o $\mathrm{Mn}$, principalmente no solo argiloso, onde ocorreu efeito positivo da calagem no seu fluxo difusivo quando na presença do resíduo de guandu ou milheto (Figura 4). Neste caso, ficou evidente que as formas complexadas por compostos orgânicos e negativamente carregadas estariam menos sujeitas às mudanças na atividade hidrogeniônica da solução do solo.

$\mathrm{O}$ aumento do fluxo difusivo do Mn para as duas resinas de troca, mesmo após a calagem, na presença de resíduos vegetais, pode estar associado à sua redução (Hue et al., 2001), que pode ser muito mais intensa no solo argiloso, quando na presença de resíduos em decomposição, que é favorecida pela correção do solo. Hue et al. (2001) verificaram um incremento do Mn na solução do solo e na parte aérea (Mn fitotóxico) da cultura da soja ao adicionar 5 e $10 \mathrm{~g} \mathrm{~kg}^{-1}$ de folhas de Vigna unguiculata L. e lodo de esgoto em solos do Hawai, em virtude do maior efeito de dissolução e de complexação desse nutriente no solo, demonstrando que a adição de compostos orgânicos ao solo cria um ambiente de redução, mesmo que temporário, diminuindo o suprimento de $\mathrm{O}_{2}$, decorrente da intensa atividade microbiana, e produzindo compostos orgânicos que podem dissolver e manter um teor elevado de Mn em solução.

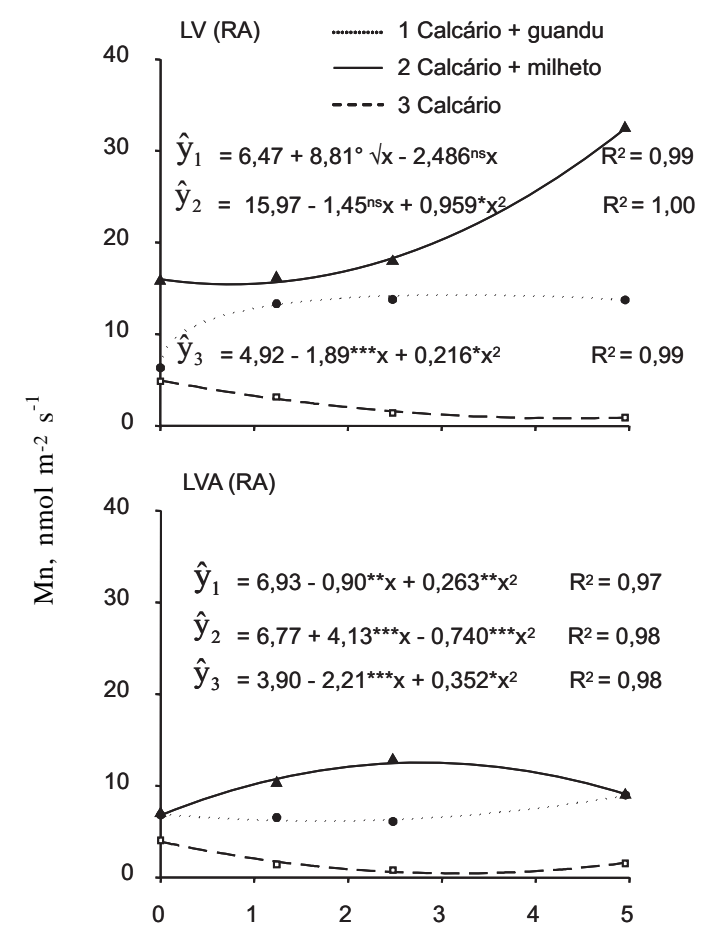

Outros trabalhos realizados com frações mais solúveis da matéria orgânica do solo, como o C orgânico solúvel em água (COSA), mostraram que a adição em diferentes solos diminuiu a sorção de $\mathrm{Cu}$ à matriz coloidal com elevação do $\mathrm{pH}$, em relação aos solos que não receberam COSA. A formação de complexos com o $\mathrm{Cu}$ (Cu-COSA) com carga líquida negativa, associada ao aumento de cargas negativas da matriz do solo em conseqüência da calagem, pode proporcionar a repulsão entre ambos, mantendo maiores teores de Cu na solução do solo (Zhou \& Wong, 2001), favorecendo o seu fluxo difusivo para as raízes das plantas.

\section{Crescimento e absorção dos micronutrientes catiônicos}

A maior produção de matéria seca de milho foi obtida no solo textura média (LVA) (Quadro 1), graças, possivelmente, ao seu maior caráter fonte do que o solo textura argilosa. Ao realizar o desdobramento da interação entre os fatores estudados, obteve-se, com a aplicação das doses crescentes de calcário, um efeito descrito pelo modelo quadrático e raiz quadrática para esta variável, sendo as maiores produtividades obtidas nas doses intermediárias do corretivo. No solo argiloso, obteve-se a máxima produção de matéria seca da parte aérea (MEF) com a dose de calcário correspondente a $1,17 \mathrm{t} \mathrm{ha}^{-1}$, nos solos que receberam somente o calcário, e a 1,14 e 2,26 t ha ${ }^{-1}$, nos solos que receberam resíduo de guandu + calcário e resíduo

CALCÁRIO, $\mathrm{t} \mathrm{ha}^{-1}$

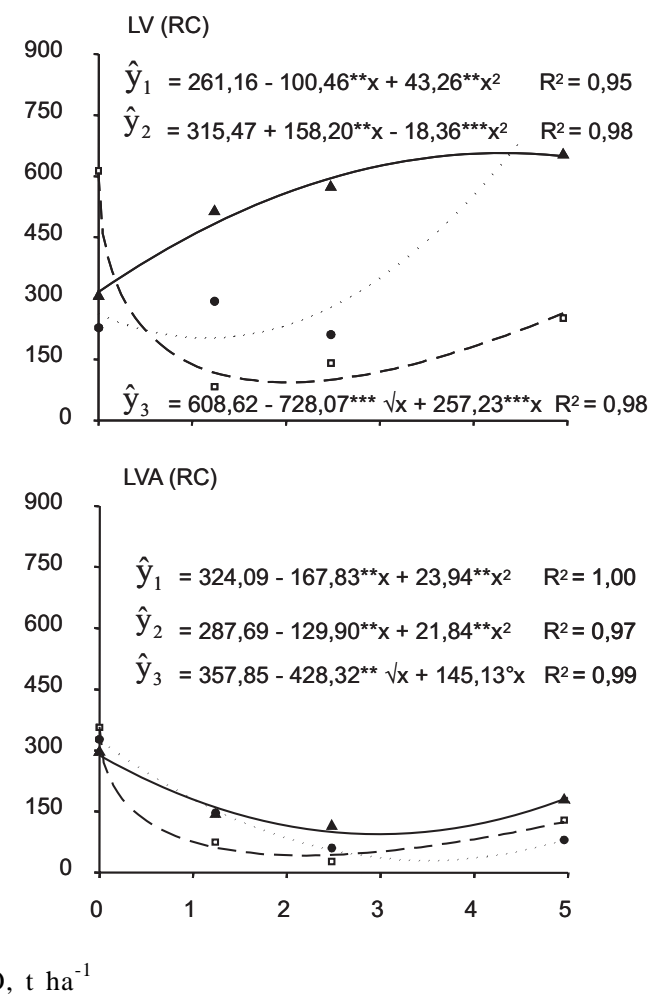

Figura 4. Fluxo difusivo de Mn para as resinas aniônicas (RA) e catiônicas (RC) em resposta à adição de resíduos vegetais de guandu ou milheto, com ou sem calagem, em solo textura média (LVA) e argilosa (LV). ${ }^{\mathrm{ns}},,^{*},{ }^{* * *},{ }^{* * *}$ : não-significativo e significativos a $\mathrm{P}<10,5,1 \mathrm{e} 0,1 \%$, respectivamente. 
de milheto + calagem, respectivamente. No solo textura média, obteve-se a MEF na dose de 3,062 t ha-1, no tratamento com calagem, e de 3,065 e 1,13 t ha ${ }^{-1}$, naqueles que receberam resíduos de guandu e milheto, respectivamente. Com isso, observou-se que a incorporação de resíduo de guandu no solo argiloso e de milheto no de textura média reduziu a dose de calcário necessária para se atingir a máxima produção de matéria seca, o que foi válido também para os teores de $\mathrm{Zn}, \mathrm{Cu}$ e Fe na parte aérea das plantas cultivadas no solo textura média e para o $\mathrm{Cu}$ no de textura argilosa. Dessa forma, as respostas em produção de matéria seca e conteúdo desses micronutrientes não dependem somente da qualidade do resíduo, mas do tipo de solo e da condição de pH a que está sendo submetido.

A interação significativa ( $\mathrm{P} \leq € 0,05)$ entre os três fatores estudados possibilitou o ajuste de equações de regressão que descrevem o crescimento de plantas de acordo com os tratamentos. Observou-se comportamento quadrático ou raiz quadrática no seu crescimento com o aumento das doses de corretivo. Este resultado pode ser associado aos baixos teores originais de Ca e $\mathrm{Mg}$ nos solos (Quadro 1), refletindo em baixos teores destes na planta, após o seu cultivo $(\mathrm{Ca}=1,2$ e 1,0 g kg-1 e $\mathrm{Mg}$ $=0,8$ e $0,7 \mathrm{~g} \mathrm{~kg}^{-1}$ com e sem a incorporação dos resíduos, no LVA, respectivamente, e de $\mathrm{Mg}=1,9 \mathrm{e}$ $1,8 \mathrm{~g} \mathrm{~kg}^{-1}$, no LV, na menor dose dos corretivos de acidez). Esses valores estão abaixo daqueles da faixa de suficiência de Ca e Mg, que é de 2,5-4,0 g kg-1 para o crescimento normal da cultura do milho (Martinez et al., 1999). Já nas doses intermediárias do corretivo, nas quais o Ca e o $\mathrm{Mg}$ não foram mais os elementos restritivos ao crescimento, obtiveram-se maiores pesos de matéria seca. Na maior dose do corretivo, pela maior elevação do $\mathrm{pH}$ dos solos, pode ter ocorrido a formação de espécies iônicas com menor solubilidade, seguido de sua precipitação (Lindsay, 1979; Barber, 1995), resultando na redução da absorção dos micronutrientes estudados (tanto na presença quanto na ausência dos resíduos vegetais), diminuindo a produção de matéria seca da planta de milho.

A presença dos resíduos vegetais também proporcionou a elevação de 0,5 a 1,0 unidade no $\mathrm{pH}$ do solo quando comparado aos tratamentos sem resíduo

Quadro 1. Matéria seca da parte aérea, teores de $\mathrm{Zn}, \mathrm{Cu}$, Fe e Mn obtidos após 25 dias de cultivo do milho, em resposta a doses de calcário $\left(0 ; 1,24 ; 2,46 ; 4,98 \mathrm{t} \mathrm{ha}^{-1}\right)$ com adição (CG e CM) ou não (C) de resíduos de guandu ou milheto no solo textura argilosa (LV) e média (LVA)

\begin{tabular}{|c|c|c|c|c|}
\hline \multirow[t]{2}{*}{ Trat. } & Equação & $\mathbf{R}^{2}$ & Equação & $\mathbf{R}^{2}$ \\
\hline & $\mathbf{L V}$ & & LVA & \\
\hline
\end{tabular}

\begin{tabular}{|c|c|c|c|c|}
\hline \multicolumn{5}{|c|}{ Matéria seca (g planta-1) } \\
\hline $\mathrm{C}$ & $\hat{\mathrm{y}}=0,573+0,423 * * \sqrt{\mathrm{x}}-0,155 * \mathrm{x}$ & 0,99 & $\hat{\mathrm{y}}=0,348+0,496 * * * \mathrm{x}-0,081 * * * \mathrm{x}^{2}$ & 0,98 \\
\hline $\mathrm{CG}$ & $\hat{\mathrm{y}}=0,378+0,747 * * * \sqrt{\mathrm{x}}-0,288 * * * \mathrm{x}$ & 0,99 & $\hat{\mathrm{y}}=0,512+0,521 * * * \mathrm{x}-0,085 * * * \mathrm{x}^{2}$ & 0,99 \\
\hline $\mathrm{CM}$ & $\hat{y}=0,693+0,122 * x-0,027 * x^{2}$ & 0,96 & $\hat{\mathrm{y}}=0,692+0,802 * * * \sqrt{\mathrm{x}}-0,314 * * * \mathrm{x}$ & 0,97 \\
\hline \multicolumn{5}{|c|}{$\mathrm{Zn}\left(\mu \mathrm{g}\right.$ planta $\left.{ }^{-1}\right)$} \\
\hline $\mathrm{C}$ & $\hat{\mathrm{y}}=\overline{\mathrm{y}}=47,333$ & & $\hat{\mathrm{y}}=29,055+44,177 * * * \mathrm{x}-8,203 * * * \mathrm{x}^{2}$ & 0,97 \\
\hline $\mathrm{CG}$ & $\hat{\mathrm{y}}=20,064+52,124 * * * \sqrt{\mathrm{x}}-24,540 * * * \mathrm{x}$ & 0,99 & $\hat{\mathrm{y}}=20,064+52,124 * * * \sqrt{\mathrm{x}}-24,540 * * * \mathrm{x}$ & 0,99 \\
\hline $\mathrm{CM}$ & $\hat{\mathrm{y}}=36,260+11,902^{\mathrm{ns}} \sqrt{\mathrm{x}-8,959} \mathrm{x}$ & 0,92 & $\hat{\mathrm{y}}=37,922+48,550 * * \sqrt{\mathrm{x}}-24,999 * * * \mathrm{x}$ & 0,98 \\
\hline \multicolumn{5}{|c|}{$\mathrm{Cu}\left(\mu \mathrm{g}\right.$ planta $\left.{ }^{-1}\right)$} \\
\hline $\mathrm{C}$ & $\hat{y}=7,817+2,273 * x-0,352^{\circ} x^{2}$ & 0,99 & $\hat{\mathrm{y}}=4,986+6,708 * * * \mathrm{x}-1,082 * * * \mathrm{x}^{2}$ & 0,99 \\
\hline $\mathrm{CG}$ & $\hat{\mathrm{y}}=5,121+7,897 * * * \sqrt{\mathrm{x}}-3,487 * * * \mathrm{x}$ & 0,96 & $\hat{\mathrm{y}}=7,595+11,456 * * * \sqrt{\mathrm{x}}-4,866 * * * \mathrm{x}$ & 0,94 \\
\hline $\mathrm{CM}$ & $\hat{\mathrm{y}}=\overline{\mathrm{y}}=8,542$ & & $\hat{\mathrm{y}}=8,936+12,206 * * * \sqrt{\mathrm{x}}-5,896 * * * \mathrm{x}$ & 0,99 \\
\hline \multicolumn{5}{|c|}{$\mathrm{Fe}\left(\mu \mathrm{g}\right.$ planta $\left.^{-1}\right)$} \\
\hline $\mathrm{C}$ & $\hat{\mathrm{y}}=\overline{\mathrm{y}}=77,452$ & & $\hat{\mathrm{y}}=41,590+44,299 * * * \mathrm{x}-7,198 * * * \mathrm{x}^{2}$ & 0,94 \\
\hline $\mathrm{CG}$ & $\hat{\mathrm{y}}=36,210+87,242 * * \sqrt{\mathrm{x}}-34,940 * * \mathrm{x}$ & 0,99 & $\hat{\mathrm{y}}=63,025+100,605 * * * \sqrt{\mathrm{x}}-41,211 * * * \mathrm{x}$ & 0,99 \\
\hline $\mathrm{CM}$ & $\hat{\mathrm{y}}=\overline{\mathrm{y}}=72,004$ & & $\hat{\mathrm{y}}=96,359+45,346 * * * \mathrm{x}-9,868 * * * \mathrm{x}^{2}$ & 0,99 \\
\hline \multicolumn{5}{|c|}{$\operatorname{Mn}\left(\mu \mathrm{g}_{\text {planta }}^{-1}\right)$} \\
\hline $\mathrm{C}$ & $\hat{\mathrm{y}}=137,940+130,892 * * * \mathrm{x}-22,726 * * * \mathrm{x}^{2}$ & 0,55 & $\hat{\mathrm{y}}=\overline{\mathrm{y}}=116,077$ & \\
\hline $\mathrm{CG}$ & $\hat{\mathrm{y}}=\overline{\mathrm{y}}=79,371$ & & $\hat{y}=72,842+56,973 * x-9,65740 * x^{2}$ & 0,88 \\
\hline
\end{tabular}

ns $,{ }^{\circ},{ }^{*},{ }^{* *},{ }^{* * *}$ : não-significativo e significativos a $\mathrm{P}<10,5,1$ e $0,1 \%$, respectivamente. 
e nas doses mais baixas do corretivo (LV: $\hat{\mathrm{y}}_{1}=6,18-$ $0,905^{*} \mathrm{x}+0,189^{*} \mathrm{x}^{2}, \mathrm{R}^{2}=0,97 ; \hat{\mathrm{y}}_{2}=6,40-0,928^{*} \mathrm{x}+$ $0,187 * x^{2}, R^{2}=0,98 ; \hat{y}_{5}=5,35+0,202 * * * x, R^{2}=0,95$; em que $\hat{y}_{1}$ calagem + guandu, $\hat{y}_{2}$ calagem + milheto e $\mathrm{y}_{5}$ calagem; LVA: $\hat{\mathrm{y}}_{1}=6,01+0,379^{*} \mathrm{x}, \mathrm{R}^{2}=0,99$; $\hat{\mathrm{y}}_{2}=6,12+0,188^{*}-0,024^{*} \mathrm{x}^{2}, \mathrm{R}^{2}=0,94 ;$ e $\hat{\mathrm{y}}_{5}=5,78$ $+0,152^{* *} \mathrm{x}, \mathrm{R}^{2}=0,98$ ), evidenciando que, em curto espaço de tempo, estes resíduos têm a capacidade de elevar o $\mathrm{pH}$ de solos ácidos. Isso decorre da precipitação do $\mathrm{Al}^{3+}$ pela elevação do $\mathrm{pH}$ pela calagem, da formação de complexos do $\mathrm{Al}$ com os ânions orgânicos e a remoção de $\mathrm{H}^{+}$decorrente da protonação de grupamentos funcionais de ácidos orgânicos liberados pelos resíduos vegetais (Franchini et al., 1999; Amaral et al., 2004).

Na presença da interação tripla entre os fatores, pôde-se verificar também que os solos, quando submetidos somente à calagem, apresentaram acréscimo linear no seu $\mathrm{pH}$ com o aumento das doses de calcário, da mesma forma que o observado para os teores de $\mathrm{Ca}$ e $\mathrm{Mg}\left(\mathrm{LV}: \hat{\mathrm{y}}_{3}=1,47+0,781^{* * *} \mathrm{x}, \mathrm{R}^{2}=1\right.$; $\hat{\mathrm{y}}_{4}=0,62+0,310^{* * *} \mathrm{x}, \mathrm{R}^{2}=1 ;$ LVA: $\hat{\mathrm{y}}_{3}=0,53+$ $1,070^{* * *} \mathrm{x}, \mathrm{R}^{2}=0,99 ; \hat{\mathrm{y}}_{4}=0,48+0,336^{* * *} \mathrm{x}, \mathrm{R}^{2}=1$,

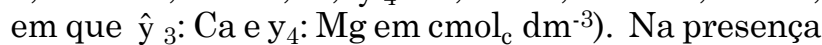
de resíduos vegetais, esse comportamento não foi verificado, pois, com o aumento das doses de calcário, o pH apresentou comportamento quadrático, com uma menor magnitude de variação entre as doses extremas: no solo argiloso, obteve-se um decréscimo do $\mathrm{pH}$ nas doses intermediárias do corretivo, e no solo textura média, um acréscimo. A natureza anfótera da reação de muitos resíduos vegetais pode fazer com que o $\mathrm{pH}$ do solo também seja reduzido, pois, segundo Miyazawa et al. (2000), o pH do solo tende ao valor de $\mathrm{pK}_{\mathrm{a}}$ da mistura de diferentes substâncias orgânicas nos resíduos vegetais e este situa-se normalmente entre 5 e 7. Em solos ácidos, a formação de ligações entre grupamentos carboxílicos $\left(\mathrm{COO}^{2-}\right)$ e íons metálicos possibilitam a elevação do seu $\mathrm{pH}$ e, em valores de $\mathrm{pH}$ mais elevados (alcalinos), os radicais amídicos ( $\mathrm{R}$ $\mathrm{CNH}_{2}-\mathrm{COO}^{2-}$ ) e fenólicos (R-OH) são os possíveis responsáveis pelo abaixamento do $\mathrm{pH}$, principalmente pelo consumo de $\mathrm{OH}^{-}$da solução do solo. Isso pode justificar o maior tamponamento do $\mathrm{pH}$ dos solos argiloso e textura média, quando se incorporou resíduo vegetal.

Finalmente, os resultados do presente estudo mostraram que práticas de manejo que resultem em aporte contínuo de material vegetal ao solo e, conseqüentemente, de compostos orgânicos que mantenham, ao longo do tempo, maior fluxo difusivo dos micronutrientes catiônicos podem favorecer o crescimento e o desenvolvimento das plantas. Possivelmente, sob condições de maior presença de resíduos vegetais, a disponibilidade reduzida de micronutrientes catiônicos, muitas vezes imposta pela calagem superficial, será menos limitante ao crescimento de plantas.

\section{CONCLUSÕES}

1. O fluxo difusivo de $\mathrm{Cu}$ e $\mathrm{Fe}$ nos solos ocorreu predominantemente sob formas aniônicas e o de Zn e Mn sob formas catiônicas.

2. O aumento das doses dos corretivos de acidez do solo reduziu o fluxo difusivo dos micronutrientes catiônicos, principalmente na forma catiônica. No entanto, a presença de resíduos vegetais aumentou o fluxo difusivo dos micronutrientes catiônicos, em ambos os solos, e minimizou o efeito redutor da calagem no fluxo difusivo de $\mathrm{Zn}, \mathrm{Cu}, \mathrm{Fe}$ e $\mathrm{Mn}$, tanto na forma aniônica como catiônica, favorecendo maior biodisponibilidade desses nutrientes para a cultura do milho.

\section{AGRADECIMENTOS}

À CAPES e ao CNPq, pelas bolsas concedidas, respectivamente, ao pós-graduando Rodinei Facco Pegoraro e aos professores Ivo Ribeiro da Silva, Roberto Ferreira de Novais e Eduardo de Sá Mendonça. Esse projeto foi parcialmente financiado pela FAPEMIG (Processo CAG 882/04) via recursos concedidos ao professor Ivo Ribeiro da Silva.

\section{LITERATURA CITADA}

ALVAREZ V., V.H. \& RIBEIRO, A.C. Calagem. In: RIBEIRO, A.C.; GUIMARÃES, P.T.G. \& ALVAREZ V., V.H., eds. Recomendação para o uso de corretivos e fertilizantes em Minas Gerais (5 ${ }^{\text {a }}$ Aproximação). Viçosa, CFSEMG, 1999. p. $43-60$

AMARAL, A.S.; ANGHINONI, I. \& DESCHAMPS, F.C. Resíduos de plantas de cobertura e mobilidade dos produtos da dissolução do calcário aplicado na superfície do solo. R. Bras. Ci. Solo, 28:115-123, 2004.

BAYER, C.; MARTIN-NETO, L.; MIELNICZUK, J. \& PAVINATO, A. Armazenamento de carbono em frações lábeis da matéria orgânica de um Latossolo Vermelho sob plantio direto. Pesq. Agropec. Bras., 39:677-683, 2004.

BARBER, S.A. Soil nutrient bioavailability: A mechanistic approach. 2.ed. New York, John Wiley \& Sons, 1995. 414p.

CAIRES, E.F. \& FONSECA, A.F. Absorção de nutrientes pela soja cultivada no sistema de plantio direto em função da calagem na superfície. Bragantia, 59:213-220, 2000.

CAIRES, E.F.; BLUM, J.; BARTH, G.; GARBUIO, F.J. \& KUSMAN, M.T. Alterações químicas do solo e resposta da soja ao calcário e gesso aplicados na implantação do sistema plantio direto. R. Bras. Ci. Solo, 27:275-286, 2003.

DUXBURY, J.M.; SMITH, M.S.; DORAN, J.W.; JORDAM, C.; SZOTT, L. \& VANCE, E. Chapter 2. Soil organic matter as a source and a sink of plant nutrient. In: COLEMAN, D.C.; OADES, J.M. \& UEHARA, G., eds. Dynamics of soil organic matter in tropical ecosystems. Honolulu, University of Hawaii, 1989. p.33-67. 
FRANCHINI, J.C.; HOFFMAN-CAMPO, C.B.; TORRES, E.; MIYAZAWA, M. \& PAVAN, M.A. Organic composition of green manure during growth and its effects on cation mobilization in an acid Oxisol. Comm. Soil Sci. Plant Anal., 34:2045-2058, 2003.

FRANCHINI, J.C.; MALAVOLTA. E.; MIYAZAWA, M. \& PAVAN, M.A. Alterações químicas em solos ácidos após a aplicação de resíduos vegetais. R. Bras. Ci. Solo, 23:533$542,1999$.

HUE, N.V.; VEGA, S. \& SILVA, J.M. Manganese toxicity in a Hawaiian Oxisol affected by soil $\mathrm{pH}$ and organic amendments. Soil Sci. Soc. Am. J., 65:153-160, 2001.

JONES, D.L.; DENNIS, P.G.; OWEN, A.G. \& van HEES, P.A.W Organic acid behavior in soils - Misconceptions and knowledge gaps. Plant Soil, 248:31-41, 2003.

JONES, D.L. Organic acids in the rhizosphere - A critical review. Plant Soil, 205:25-44, 1998.

KORTE, N.E.; SKOPP, J.; FULLER, W.H.; NIEBLA, E.E. \& ALESII, B.A. Trace element movement in soils: Influence of soil physical and chemical properties. Soil Sci., 122:350359, 1976.

LINDSAY, W. Chemical equilibria in soil. New York: John Wiley \& Sons, 1979. 449p.

MARTINEZ, H.E.P.; CARVALHO, J.G. \& SOUZA, R.B. Diagnose foliar. In: RIBEIRO, A. C.; GUIMARÃES, P.T.G. \& ALVAREZ V., V.H., eds. Recomendação para o uso de corretivos e fertilizantes em Minas Gerais $\left(5^{\text {a }}\right.$ Aproximação). Viçosa, CFSEMG, 1999. p.43-60.

MIYAZAWA, M; FRANCHINI, J.C. \& PAVAN, M.A. Neutralização da acidez do perfil do solo por resíduos vegetais. www.potafos.org/brasil.nsf (15/01/03). Encarte Técnico, 2000. 15p. (Informações Agronômicas, 92)

NOVAIS, R.F.; NEVES, J.C.L. \& BARROS, N.F. Ensaio em ambiente controlado. In: OLIVEIRA, A.J.; GARRIDO, W.E.; ARAUJO, J.D. \& LOURENCO, S., eds. Métodos de pesquisa em fertilidade do solo. Brasília, Embrapa-SEA, 1991. p.189-254.

NUNES, F.N.; NOVAIS, R.F.; SILVA, I.R.; GEBRIM, F.O. \& SÃO JOSÉ, J.F.B. Fluxo difusivo de ferro em solos sob influência de doses de fósforo e de níveis de acidez e umidade. R. Bras. Ci. Solo, 28:423-429, 2004.

OLIVEIRA, T.K.; CARVALHO, G.J. \& MORAES, R.N.S. Plantas de cobertura e seus efeitos sobre o feijoeiro em plantio direto. Pesq. Agropec. Bras., 37:1079-1087, 2002.
OLIVEIRA, M.F.G.; NOVAIS, R.F.; NEVES, J.C.L.; ALVES, V.M.C. \& VASCONCELLOS, C.A. Fluxo difusivo de zinco em amostras de solo influenciado por textura, íon acompanhante e pH do solo. R. Bras. Ci. Solo, 23:609-615, 1999.

PEGORARO, R.F. Fluxo difusivo de micronutrientes catiônicos em resposta à adição de compostos orgânicos ao solo. Viçosa, MG, Universidade Federal de Viçosa, 2003. 78p. (Tese de Mestrado)

RENELLA, G.; LANDI, L. \& NANNIPIERI, P. Degradation of low molecular weight organic acids complexed with heavy metals in soil. Geoderma, 122:311-315, 2004.

RHOTON, F.E. Influence of time on soil response to no-till practices. Soil Sci. Soc. Am. J., 64:700-709, 2000.

SÁ, J.C.M.; CERRI, C.C.; DICK, W.A.; LAL, R.; VENSKE FILHO, S.P.; PICCOLO, M.C. \& FEIGL, B.E. Organic matter dynamics and carbon sequestration rates for a tillage chronosequence in a Brazilian Oxisol. Soil Sci. Soc. Am. J., 65:1486-1499, 2001.

SANDERS, J.R. The effect of $\mathrm{pH}$ on the total and free ionic concentrations of manganese, zinc and cobalt in soil solution. J. Soil Sci., 34:315-323, 1983.

SILVA, I.R.; SMYTH, T.J.; RAPER, C.D.; CARTER, T.E. \& RUFTY, T.W. Differential aluminum tolerance in soybean: An evaluation of the role of organic acids Physiol. Plant., 112:200-210, 2001.

STROBEL, B.W. Influence of vegetation on low-molecularweight carboxylic acids in soil solution - A review. Geoderma, 99:169-198, 2001.

TEMMINGHOFF, E.J.M.; van der ZEE, S.E.A.T.M. \& HAAN, F.A.M. Effects of dissolved organic matter on the mobility of copper in a contaminated sandy soil. Eur. J. Soil Sci., 49:617-628, 1998.

VULKAN, R.; MINGELGRIN, U.; BEN-ASHER, J. \& FRENKEL, H. Copper and zinc speciation in the solution of a soil-sludge mixture. J Environ. Quality, 31:193-203, 2002 .

ZHOU, L.X. \& WONG, J.W.C. Effect of dissolved organic matter from sludge and sludge compost on soil copper sorption. J. Environ. Quality, 30:878-883, 2001.

van HEES, P.A.W.; VINOGRADOFF, A.C.; EDWARDS, A.C.; GODBOLD, D.L. \& JONES, D.L. Low molecular weight organic acid adsorption in forest soils: Effects on soil solution concentrations and biodegradation rates. Soil Biol. Biochem., 35:1015-1026, 2003. 